\title{
Stationary axisymmetric SU(2) Einstein-Yang-Mills fields with restricted circularity conditions are Abelian
}

\author{
F. J. Chinea and F. Navarro-Lérida \\ Departamento de Física Teórica II, Ciencias Físicas, Universidad Complutense de Madrid, E-28040 Madrid, Spain
}

(Received 16 August 2001; published 12 February 2002)

\begin{abstract}
In this paper we prove that in a stationary axisymmetric SU(2) Einstein-Yang-Mills theory the most reasonable circularity conditions that can be considered for the Yang-Mills fields imply in fact that the field is of embedded Abelian type, or else that the metric is not asymptotically flat.
\end{abstract}

DOI: 10.1103/PhysRevD.65.064010

PACS number(s): 04.20.Cv, 12.10.-g, 12.15.-y

Since the discovery of the existence of regular solutions in the SU(2) Einstein-Yang-Mills (EYM) theory [1] a lot of effort trying to find new interesting non-Abelian solutions has been expended and new solutions (most of them in a numerical form) have been found in the presence of symmetries (spherical and axisymmetric cases) and for static and stationary spacetimes (see references in [2]). In this paper we will concentrate on the stationary axisymmetric SU(2) EYM theory. When working with stationary axisymmetric YangMills (YM) fields, most of the (numerical) known solutions make use of the same ansatz: the one introduced by Manton [3] and Rebbi and Rossi [4]. However, this ansatz prevents the use of Weyl's coordinates for spacetime, as opposed to what can be done in Einstein-Maxwell theory. The possibility of using Weyl's coordinates would simplify considerably the Einstein equations but the Ansätze considered up to now in this direction have (numerically) been shown to be incompatible with non-Abelianity and asymptotic flatness. Nevertheless, there seems to be no general argument against the possibility of the existence of an ansatz suitable to merge asymptotic flatness and the non-Abelian nature with the use of Weyl's coordinates for the metric. Here, we clarify this point by proving rigorously that a rather natural condition [see Eq. (16)] for fulfilling that ansatz cannot in fact be imposed, as a non-Abelian solution for the stationary axisymmetric SU(2) EYM equations with the appropriate asymptotically flat behavior cannot exist.

In order to show this result, let $\boldsymbol{\xi}$ and $\boldsymbol{\eta}$ be the Killing vectors that generate the Abelian group $G_{2}$ of isometries of the stationary axially symmetric spacetime, $\boldsymbol{\xi}$ being a timelike vector field and $\boldsymbol{\eta}$ being a spacelike one with compact periodic trajectories. Owing to the fact that both of them commute, we are able to choose adapted coordinates, say $t$ and $\phi$, such that $\boldsymbol{\xi}=\partial_{t}$ and $\boldsymbol{\eta}=\partial_{\phi}$. We will also assume that the elementary flatness condition for $\boldsymbol{\eta}$ is satisfied so that the axis is a regular two-dimensional submanifold of the spacetime. We further impose on the stationary axisymmetric spacetime that it admits 2-spaces orthogonal to the group orbits, that is to say, that

$$
\xi \wedge \eta \wedge d \xi=\xi \wedge \eta \wedge d \eta=0
$$

holds, where $\xi(\eta)$ is the 1-form corresponding to the vector field $\boldsymbol{\xi}(\boldsymbol{\eta})$. Now, if one assumes that the metric contains the axis (or, at least, one of its points), the (Ricci-) circularity theorem $[5,6]$ states that Eq. (1) is equivalent to

$$
\xi \wedge \eta \wedge R(\xi)=\xi \wedge \eta \wedge R(\boldsymbol{\eta})=0,
$$

where $R(\boldsymbol{v}) \equiv R_{\mu \nu} v^{\mu} d x^{\nu}, R_{\mu \nu}$ being the Ricci tensor. With these assumptions the metric can be written in the LewisPapapetrou form $[7,8]$ :

$$
d s^{2}=-f(d t-\omega d \phi)^{2}+f^{-1}\left[e^{2 \gamma}\left(d \rho^{2}+d z^{2}\right)+W^{2} d \phi^{2}\right],
$$

where $f, \omega, \gamma$, and $W$ are functions of the $\rho$ and $z$ coordinates.

Let us now suppose that the matter content is given by non-Abelian SU(2) gauge fields coupled to gravity. We will use the following expression for the YM field $F$ :

$$
F=d A+A \wedge A,
$$

where the YM potential $A$ is an su(2)-valued 1-form, which satisfies

$$
A^{\dagger}=-A, \quad \operatorname{tr} A=0 .
$$

In components

$$
F_{\mu \nu}=A_{\nu, \mu}-A_{\mu, \nu}+\left[A_{\mu}, A_{\nu}\right]
$$

where a comma followed by an index denotes a partial derivative with respect to the corresponding coordinate. The EYM equations read

$$
\begin{aligned}
G_{\mu \nu} & =8 \pi G T_{\mu \nu}, \\
D_{\mu} F^{\mu \nu} & =0
\end{aligned}
$$

where $G_{\mu \nu}$ is the Einstein tensor; here and in the following we take $c=1$. The YM equations (8) can also be conveniently expressed as

$$
d^{*} F+A \wedge * F-* F \wedge A=0,
$$

where $* F$ denotes the Hodge dual of $F$. The energymomentum tensor, $T_{\mu \nu}$, and the gauge-covariant derivative, $D_{\mu}$, are defined as

$$
\begin{aligned}
T_{\mu \nu} & \equiv \frac{1}{2 \pi} \operatorname{tr}\left\{-F_{\mu \sigma} F_{\nu}{ }^{\sigma}+\frac{1}{4} g_{\mu \nu} F_{\alpha \beta} F^{\alpha \beta}\right\}, \\
D_{\mu} & \equiv \nabla_{\mu}+\left[A_{\mu}, \cdot\right],
\end{aligned}
$$


where $\nabla_{\mu}$ denotes the covariant derivative. For a review of EYM fields, see [2].

By using the Einstein equations (7), conditions (2) can be rewritten as

$$
\xi \wedge \eta \wedge T(\xi)=\xi \wedge \eta \wedge T(\boldsymbol{\eta})=0,
$$

which is just the same as asking $T_{t \rho}, T_{t z}, T_{\phi \rho}$, and $T_{\phi z}$ to vanish. Following Heusler and Straumann [9] and Heusler [10], one can write the Ricci-circularity conditions in a more compact form:

$$
\begin{aligned}
& \operatorname{tr}\left\{F(\boldsymbol{\xi}, \boldsymbol{\eta}) B_{\xi}+{ }^{*} F(\boldsymbol{\xi}, \boldsymbol{\eta}) E_{\boldsymbol{\xi}}\right\}=0, \\
& \operatorname{tr}\left\{F(\boldsymbol{\xi}, \boldsymbol{\eta}) B_{\boldsymbol{\eta}}+{ }^{*} F(\boldsymbol{\xi}, \boldsymbol{\eta}) E_{\boldsymbol{\eta}}\right\}=0,
\end{aligned}
$$

where $E_{\boldsymbol{v}}$ and $B_{\boldsymbol{v}}$ stand for $-i_{\boldsymbol{v}} F$ and $i_{\boldsymbol{v}}{ }^{*} F$, respectively, $i_{\boldsymbol{v}}$ being the inner product (see [10] for definitions). These conditions are valid for $\mathrm{SU}(\mathrm{N})$ in general.

The next step is to impose symmetries on the YM fields. Following Bergmann and Flaherty [11] and Forgàcs and Manton [12], and taking into account that $\boldsymbol{\xi}$ and $\boldsymbol{\eta}$ commute, it is possible to use part of the gauge freedom in order to write the symmetries on the YM potentials as

$$
\mathcal{L}_{\xi} A_{\mu}=\mathcal{L}_{\eta} A_{\mu}=0, \quad \text { i.e., } \quad A_{\mu}=A_{\mu}(\rho, z) .
$$

Some gauge freedom still remains, allowing us to perform transformations which depend on $\rho$ and $z$ only, if necessary.

Looking at Eqs. (13) and (14), which represent four different constraints, one could ask oneself if they might be a consequence of the EYM equations plus the symmetry conditions. That is true for the electromagnetic case, because the relations

$$
\xi \wedge \eta \wedge F=\xi \wedge \eta \wedge * F=0
$$

follow from the Maxwell equations and the fact that $A$ $=A(\rho, z)$. However, as claimed in [9], in a non-Abelian case there are no known general arguments derived from EYM equations and symmetry conditions that establish $F(\boldsymbol{\xi}, \boldsymbol{\eta})$ $=* F(\boldsymbol{\xi}, \boldsymbol{\eta})=0$. Nevertheless, these appear to be the most reasonable assumptions one can put forward in order to satisfy equations (13) and (14). It should be noticed that this ansatz for the YM fields is conserved under gauge transformations, as it is imposed on the YM fields instead of on the YM potentials.

In the following, we will assume the restricted circularity conditions $F(\boldsymbol{\xi}, \boldsymbol{\eta})={ }^{*} F(\boldsymbol{\xi}, \boldsymbol{\eta})=0$ (in coordinates, $F_{t \phi}=F_{\rho z}$ $=0$ ) for stationary axisymmetric $\mathrm{SU}(2)$ gauge fields, and we will prove that these natural assumptions give rise to embedded Abelian cases or to non-asymptotically flat spacetimes. Using $F_{t \phi}=F_{\rho z}=0$ in Eq. (7), it is very easy to see that $W$ has to be harmonic, i.e.,

$$
W_{, \rho \rho}+W_{, z z}=0
$$

For this reason it is possible to perform a coordinate transformation such that $W=\rho$ (Weyl's coordinates), so that the metric may be written as

$$
d s^{2}=-f(d t-\omega d \phi)^{2}+f^{-1}\left[e^{2 \gamma}\left(d \rho^{2}+d z^{2}\right)+\rho^{2} d \phi^{2}\right] .
$$

In these coordinates it can be shown that the Einstein equations (7) reduce to the following two equations where neither $\gamma$ nor its derivatives appear, plus other equations for $\gamma$, which will not be relevant in what follows:

$$
\begin{aligned}
\rho^{2} f & \nabla^{2} f-\rho^{2}(\nabla f)^{2}+f^{4}(\nabla \omega)^{2} \\
& =-4 G f \operatorname{tr}\left\{f^{2}\left(\omega F_{t \rho}-F_{\rho \phi}\right)^{2}+f^{2}\left(\omega F_{t z}-F_{z \phi}\right)^{2}\right. \\
& \left.+\rho^{2}\left(F_{t \rho}^{2}+F_{t z}^{2}\right)\right\}, \\
\nabla \cdot\left(\rho^{-2} f \nabla \omega\right) & =-8 G \rho^{-2} f \operatorname{tr}\left\{F_{t \rho} F_{\rho \phi}+F_{t z} F_{z \phi}-\omega\left(F_{t \rho}^{2}+F_{t z}^{2}\right)\right\},
\end{aligned}
$$

where $\nabla$ represents the 3 -dimensional flat-space nabla operator in cylindrical coordinates $(\rho, z, \phi)$.

Let us now concentrate on the YM equations and more concretely on the $\rho$ and $z$ components. In order to simplify them we use the constraint $F_{t \phi}=0$. Because of our choice of a gauge where $A$ depends on $\rho$ and $z$ only and the fact that the gauge group is $\mathrm{SU}(2)$, the above restriction leads to two possibilities: $A_{\phi}=0$ or $A_{t}=\lambda A_{\phi}$, with $\lambda=\lambda(\rho, z)$ a scalar function.

For the first one it is easy to prove that every component of $F$ has to be proportional to $A_{t}$. We shall show immediately that this leads to an embedded Abelian field [recall that, by definition, an embedded Abelian field is one such that its potential $A$ satisfies $A=\beta T_{0}$, where $\beta$ is a scalar 1-form and $T_{0}$ a constant element $\left(d T_{0}=0\right)$ in the Lie algebra of the gauge field; the YM equations are then equivalent to the Maxwell equations for the potential $\left.\beta, d^{*}(d \beta)=0\right]$. The proof proceeds as follows: If all components of $F$ commute with $A_{t}$, then they are all proportional to a common element in $\operatorname{su}(2)$, and $\left[F_{\mu \nu}, F_{\alpha \beta}\right]=0$, for all indices $\mu, \nu, \alpha, \beta$. This can be expressed by the statement

$$
F=\sigma T
$$

where $\sigma$ is a scalar 2-form and $T$ is a (in general, coordinatedependent) 0 -form with values in the Lie algebra su(2). By imposing the Bianchi identity $d F+A \wedge F-F \wedge A=0$ on $F$, we get

$$
0=d \sigma T+\sigma \wedge(d T+[A, T]) .
$$

We deal successively with two possible cases: either $d T$ $+[A, T]$ is proportional to $T$ or they are independent. In the first case, $d T+[A, T]=\alpha T$, where $\alpha$ is a scalar 1 -form. By exterior differentiation of this equation, we get $d \alpha=0$, thus giving locally $\alpha=d h$ for a certain function $h$. Substituting this in Eq. (22), we get $0=d \sigma+\sigma \wedge d h$, so that $d\left(e^{h} \sigma\right)=0$. Then, there will exist locally a 1 -form $\beta$ such that

$$
\sigma=e^{-h} d \beta
$$


The YM equation (9) for $F$ yields $0=e^{-h} d(* d \beta) T$, i.e., $d^{*}(d \beta)=0$, thus showing that the physical content of a YM field satisfying Eq. (21) is simply that of a Maxwell field. By defining $\widetilde{T} \equiv e^{-h} T$, we get

$$
\begin{aligned}
F & =d \beta \widetilde{T}, \\
d \widetilde{T} & =\widetilde{T} \wedge A-A \wedge \widetilde{T}, \\
d(* d \beta) & =0 .
\end{aligned}
$$

In the case where $T$ and $d T+[A, T]$ are independent, we get $d \sigma=0$ and $d T+[A, T]=0$. There will exist locally $\beta$ such that $\sigma=d \beta$. The resulting equations are similar to Eqs. (24)(26):

$$
\begin{aligned}
F & =d \beta T, \\
d T & =T \wedge A-A \wedge T, \\
d(* d \beta) & =0 .
\end{aligned}
$$

We shall now conclude from Eqs. (27)-(29) that the gaugeinvariant condition (21) is equivalent to the standard definition of embedded Abelian fields [similar considerations apply to the formally identical equations (24)-(26)]: By defining

$$
C \equiv A-\beta T,
$$

and substituting $A=\beta T+C$ in Eq. (27), we conclude

$$
d C+C \wedge C=0,
$$

so that $C$ is pure gauge [locally, $C=S^{-1} d S$, for an SU(2)valued 0 -form $S$ ]. Thus, $A=\beta T+S^{-1} d S$. By substituting this expression for $A$ in Eq. (28), we get $d\left(S T S^{-1}\right)=0$, so that $S T S^{-1}=T_{0}$, for a certain $T_{0}$, with $d T_{0}=0$. In conclusion,

$$
A=S^{-1} \beta T_{0} S+S^{-1} d S,
$$

so that $A$ is just a gauge transform of

$$
A_{0}=\beta T_{0} .
$$

[Please notice that the gauge transformation $S$ may be chosen to be independent of $t$ and $\phi$, thus leaving condition (15) invariant.]

Let us consider more closely the other option:

$$
A_{t}=\lambda A_{\phi} .
$$

When one substitutes Eq. (34) into the $\rho$ and $z$ components of Eq. (8) the following relations are obtained:

$$
\begin{aligned}
& (\lambda f \omega+\rho \lambda+f)(\lambda f \omega-\rho \lambda+f)\left[A_{\phi}, F_{\phi \rho}\right]=0, \\
& (\lambda f \omega+\rho \lambda+f)(\lambda f \omega-\rho \lambda+f)\left[A_{\phi}, F_{\phi z}\right]=0 .
\end{aligned}
$$

There are three possible choices. If the commutators vanish, we apply the result just proved above, and we obtain again an embedded Abelian solution, as every component of $F$ can be shown to be proportional to $A_{\phi}$. As for the two other possibilities, they essentially reduce to the same one because they are related by means of a reversal of the sense of rotation $(\lambda \rightarrow-\lambda, \omega \rightarrow-\omega)$. For that reason, we can choose one of them, our result being valid for the other one, too. As a consequence, the form for the function of proportionality between $A_{t}$ and $A_{\phi}$ reads

$$
\lambda=\frac{f}{\rho-f \omega} .
$$

There still remain the two other components of the YM equations, namely, the $t$ and $\phi$ components. Using on the previously mentioned equations the relations

$$
\begin{gathered}
F^{\rho t}+\frac{1}{\lambda} F^{\rho \phi}=-\frac{f}{\rho e^{2 \gamma}} \frac{\lambda_{, \rho}}{\lambda} A_{\phi}, \\
F^{z t}+\frac{1}{\lambda} F^{z \phi}=-\frac{f}{\rho e^{2 \gamma}} \frac{\lambda_{, z}}{\lambda} A_{\phi},
\end{gathered}
$$

derived from Eq. (34), one obtains the following equation:

$$
\left\{\lambda_{, \rho \rho}+\lambda_{, z z}-\frac{\rho+f \omega}{\rho \lambda}\left(\lambda_{, \rho}^{2}+\lambda_{, z}^{2}\right)\right\} A_{\phi}=0 .
$$

As said before, the case with $A_{\phi}=0$ is an embedded Abelian one, so we will concentrate on the other possibility:

$$
\lambda_{, \rho \rho}+\lambda_{, z z}-\frac{\rho+f \omega}{\rho \lambda}\left(\lambda_{, \rho}^{2}+\lambda_{, z}^{2}\right)=0 .
$$

The second-order derivatives in Eq. (41) may be substituted by using Eq. (37) and a combination of the Einstein field equations (19) and (20) [to be more precise, we use the field equation corresponding to $-(\rho-f \omega)^{2} G_{t t}+2 f(\rho$ $\left.-f \omega) G_{t \phi}-f^{2} G_{\phi \phi}\right]$. The resulting equation reads

$$
\rho f_{, \rho}+f^{2} \omega_{, \rho}-f=-4 G \frac{f^{2}}{\lambda^{2}}\left(\lambda_{, \rho}^{2}+\lambda_{, z}^{2}\right) \operatorname{tr}\left(A_{\phi}^{2}\right) .
$$

From Eq. (42) we see that the left-hand side has to be nonnegative [recall that $\operatorname{tr}\left(A_{\phi}^{2}\right)<0$ ], but in that case the asymptotically flat condition cannot hold. To prove that, one only has to introduce in Eq. (42) the asymptotic behavior of $f$ and $\omega$

$$
\begin{aligned}
& f \rightarrow 1-\frac{2 M}{r}+O\left(\frac{1}{r^{2}}\right), \\
& \omega \rightarrow-\frac{2 J}{r} \sin ^{2} \theta+O\left(\frac{1}{r^{2}}\right),
\end{aligned}
$$


where $r$ and $\theta$ are spherical coordinates related to $\rho$ and $z$ as $r=\sqrt{\rho^{2}+z^{2}}$ and $\theta=\arctan (\rho / z)$, and $M$ and $J$ are constants. When this is done, the leading term of the left-hand side goes like -1 , yielding a contradiction.

Thus, the only case which is not essentially Abelian has to be nonasymptotically flat, which makes it unacceptable. We have proved this result for an SU(2) EYM theory. Our method depends on the fact that for $\mathrm{SU}(2)$ a vanishing commutator of two quantities in the corresponding Lie algebra implies that either one of them vanishes, or that a relation such as Eq. (34) holds. However, this is not true for $\mathrm{SU}(\mathrm{N})$ in general, because if $\mathrm{N}$ is greater than two, it is possible to find two-dimensional Abelian subalgebras in the associated Lie algebra. Therefore, the procedure followed here cannot be generalized to $\mathrm{SU}(\mathrm{N})$ in general.

The present work has been supported in part by DGICYT Project PB98-0772; F.N.L. is supported by Ministerio de Educación (Spain). The authors wish to thank L. FernándezJambrina, L. M. González-Romero, and M. J. Pareja for discussions.
[1] R. Bartnik and J. McKinnon, Phys. Rev. Lett. 61, 141 (1988).

[2] M.S. Volkov and D.V. Gal'tsov, Phys. Rep. 319, 1 (1999).

[3] N.S. Manton, Nucl. Phys. B135, 319 (1978).

[4] C. Rebbi and P. Rossi, Phys. Rev. D 22, 2010 (1980).

[5] W. Kundt and M. Trümper, Ann. Phys. (Leipzig) 192, 414 (1966).

[6] B. Carter, J. Math. Phys. 10, 70 (1969).

[7] T. Lewis, Proc. R. Soc. London A136, 176 (1932).

[8] A. Papapetrou, Ann. Inst. Henri Poincaré, Sect. A 4, 83 (1966).
[9] M. Heusler and N. Straumann, Class. Quantum Grav. 10, 1299 (1993).

[10] M. Heusler, Black Hole Uniqueness Theorems (Cambridge University Press, Cambridge, England, 1996).

[11] P.G. Bergmann and F.J. Flaherty, J. Math. Phys. 19, 212 (1978).

[12] P. Forgàcs and N.S. Manton, Commun. Math. Phys. 72, 15 (1980). 